\title{
LC-MS screening of poly- and perfluoroalkyl substances in contaminated soil by Kendrick mass analysis
}

\author{
Boris Bugsel $^{1} \cdot$ Christian $_{\text {Zwiener }^{1}}$ (D) \\ Received: 2 October 2019 / Revised: 6 December 2019 / Accepted: 16 December 2019 / Published online: 9 January 2020 \\ (C) The Author(s) 2020
}

\begin{abstract}
The application of contaminated paper sludge on arable land in southwest Germany caused the occurrence of a broad range of poly- and perfluoroalkyl substances (PFASs) on soil. Recently, the dead-end transformation products (TPs) perfluorooctanoic acid and perfluorooctanesulfonic acid were detected in groundwater and drinking water. The precursors and other transformation products mostly remained unknown. Therefore, HRMS screening by Kendrick mass analysis and assignment of homologous series in combination with suspect screening were applied to identify original PFASs and their TPs in four different soil samples from sites where contaminated paper sludge was applied. In total, twelve compound classes comprising more than 61 PFASs could be fully or tentatively identified. The data reveal that contamination mainly originates from polyfluorinated dialkylated phosphate esters (from 4:2/6:2 to 12:2/14:2), $N$-ethyl perfluorooctane sulfonamide ethanol-based phosphate diesters (only $\mathrm{C}_{8} /$ $\mathrm{C}_{8}$ ) and transformation products of these precursors. Contamination patterns can be attributed to PFASs used for paper impregnation and can vary slightly from site to site.
\end{abstract}

Keywords Poly- and perfluoroalkyl substances · High-resolution mass spectrometry · Screening · Kendrick mass · Soil · Contamination

\begin{tabular}{|c|c|c|c|}
\hline \multicolumn{2}{|c|}{ Abbreviations } & EtFASAA & $N$-Ethylperfluoro-1-alkanesulfonamidoacetic \\
\hline AIF & All-ion fragmentation & & acid \\
\hline $\mathrm{Da}$ & Dalton & EtFOSAA & $N$-Ethylperfluoro-1-octanesulfonamidoacetic \\
\hline diPAP & Polyfluorinated dialkylated phosphate ester & & acid \\
\hline \multirow[t]{2}{*}{ diSAmPAP } & $N$-Ethyl perfluorooctane sulfonamide ethanol- & FASA & Perfluoroalkanesulfonamide \\
\hline & based phosphate diester & FOSA & Perfluorooctanesulfonamide \\
\hline EIC & Extracted ion chromatogram & FTOH & Fluorotelomer alcohol \\
\hline ESI & Electrospray ionization & FTSA & Fluorotelomer sulfonic acid \\
\hline \multirow{2}{*}{\multicolumn{2}{|c|}{ EtFASA }} & FTUCA & Fluorotelomer unsaturated carboxylic acid \\
\hline & & GenX & Ammonium $2,3,3,3-$ tetrafluoro- \\
\hline \multirow{2}{*}{\multicolumn{2}{|c|}{$\begin{array}{l}\text { Published in the topical collection Persistent and Mobile Organic } \\
\text { Compounds - An Environmental Challenge with guest editors Torsten C. } \\
\text { Schmidt, Thomas P. Knepper, and Thorsten Reemtsma. }\end{array}$}} & HRMS & $\begin{array}{l}\text { High-resolution mass spectrometry } \\
\text { Highe }\end{array}$ \\
\hline & & HS & Homologous series \\
\hline \multirow{3}{*}{\multicolumn{2}{|c|}{$\begin{array}{l}\text { Electronic supplementary material The online version of this article } \\
\text { (https://doi.org/10.1007/s00216-019-02358-0) contains supplementary } \\
\text { material, which is available to authorized users. }\end{array}$}} & KM & Kendrick mass \\
\hline & & KMD & Kendrick mass defect \\
\hline & & $\mathrm{LC}$ & Liquid chromatography \\
\hline$\triangle$ Christian 2 & wiener & LTZ & $\begin{array}{l}\text { Landwirtschaftliches Technologiezentrum } \\
\text { Augustenberg }\end{array}$ \\
\hline Christian.Z & wiener@uni-tuebingen.de & MeFASA & $N$-Methyl perfluoroalkanesulfonamide \\
\hline \multirow{3}{*}{\multicolumn{2}{|c|}{$\begin{array}{l}\text { Environmental Analytical Chemistry, Center for Applied } \\
\text { Geoscience, University of Tübingen, Hölderlinstraße 12, } \\
72074 \text { Tübingen, Germany }\end{array}$}} & $\mathrm{MeOH}$ & Methanol \\
\hline & & MFE & Molecular feature extraction \\
\hline & & $\mathrm{NH}_{4} \mathrm{Ac}$ & Ammonium acetate \\
\hline
\end{tabular}




$\begin{array}{ll}\text { OM } & \text { Observed mass } \\ \text { PFAAs } & \text { Perfluoroalkyl acids } \\ \text { PFASs } & \text { Poly- and perfluoroalkyl substances } \\ \text { PFCAs } & \text { Perfluorocarboxylic acids } \\ \text { PFHpA } & \text { Perfluoroheptanoic acid } \\ \text { PFNA } & \text { Perfluorononanoic acid } \\ \text { PFOA } & \text { Perfluorooctanoic acid } \\ \text { PFOS } & \text { Perfluorooctanesulfonic acid } \\ \text { PFPIA } & \text { Perfluorinated phosphonic acid } \\ \text { PFSAs } & \text { Perfluorosulfonic acids } \\ \text { PP } & \text { Polypropylene } \\ \text { rcf } & \text { Relative centrifugal force } \\ \text { RT } & \text { Retention time } \\ \text { TP } & \text { Transformation product } \\ \text { triPAP } & \text { Polyfluorinated trialkylated phosphate ester }\end{array}$

\section{Introduction}

Poly- and perfluorinated alkylated substances (PFASs) are anthropogenic compounds with an increasing environmental significance [1]. They comprise a family of more than 4700 single compounds [2]. Due to their unique, both water- and grease-repelling properties, their fields of applications are textiles such as clothing and carpets [3], nonstick cookware [4], food packaging [5,6], cosmetics [7], and firefighting foams $[8,9]$. The widespread use of PFASs has led to a global distribution in biota [10], air [11], soil [12], and water where they were even proposed as novel tracers [13]. While the characteristics of PFASs render them desirable for the industry, their negative impacts on both environment and health are beyond question. Two of the best studied PFASs, perfluorooctanoic acid (PFOA) and perfluorooctanesulfonic acid (PFOS), accumulate in the liver, lung, and kidney of rats and are proposed to be a big threat to humans and biota $[14,15]$. Their major manufacturer $3 \mathrm{M}$ has hence phased out the production of these two substances between 2000 and 2002 [1], yet other PFASs such as ammonium 2,3,3,3-tetrafluoro2-(heptafluoropropoxy)-propanoate (GenX) have replaced these compounds [16]. Studies performed by Gomis et al. [17] and Sun et al. [18] even indicate a higher toxicity and a lower sorption on activated carbon for GenX than for PFOA, highlighting the importance of both legacy and novel PFASs.

Liquid chromatography coupled to mass spectrometry has earlier been used in the identification and determination of PFASs [5, 8, 19-22]. The use of high-resolution mass spectrometry (HRMS) reveals valuable accurate mass information which can be used in the identification of unknown contaminants. The nature of PFASs typically is their occurrence in multiple homologues, a beneficial characteristic in their identification. Coupling HRMS with homologous series (HS) detection has a variety of useful applications such as the detection of surfactants or polymers $[23,24]$ and PFASs $[25,26]$.
In summer 2013, a routine sampling has revealed PFAS contamination of a drinking water well near Rastatt (BadenWürttemberg, Germany) [27]. Further research showed that soil contamination on agricultural land has led to groundwater pollution. Compost mixed with paper sludge containing PFASs was applied to about $7.8 \mathrm{Mio}^{2}$ agricultural soil $[28,29]$. A large variety of both anionic and non-ionic PFASs is known to be applied to food packaging paper and board in order to grant oil and water repellency [6]. Schaider et al. [30] found that $46 \%$ out of roughly 400 food contact papers showed detectable levels of fluorinated substances. Typically, these PFAS paper impregnation agents can contain multiple alkyl chains and functional groups [6]. Lab experiments revealed that the original PFAS paper impregnation products (precursors) are slowly degrading to short single-chain perfluoroalkyl acids (PFAAs) such as perfluorocarboxylic acids (PFCAs) and perfluorosulfonic acids (PFSAs) [31, 32]. The transformation products (TPs) are more mobile, migrate to groundwater, and can be taken up by plants $[33,34]$. To evaluate the exposure of the population which received contaminated drinking water, blood tests were conducted. Results of the contaminated area showed increased blood levels of PFASs of the exposed people (e.g., $15.7 \mu \mathrm{g} / \mathrm{L}$ PFOA) compared to controls $(1.7 \mu \mathrm{g} / \mathrm{L}$ PFOA) [35]. In other regions, PFOA blood concentrations of exposed people are also about 10 -fold higher than those of control population [36].

In the present case study, information on the identity and amount of technical products used and discharged is very limited. These precursors are typically not included in target methods. The aim of the present work hence is the characterization and identification of the original contamination and its TPs. Here, we used a non-target approach with LC-highresolution mass spectrometry and Kendrick mass analysis.

\section{Materials and methods}

\section{Chemicals and reagents}

Optima LC-MS grade methanol (MeOH), ammonium acetate $\left(\mathrm{NH}_{4} \mathrm{Ac}\right)$, and water were purchased from Fisher Scientific. Authentic reference standards of sodium $(1 \mathrm{H}, 1 \mathrm{H}, 2 \mathrm{H}, 2 \mathrm{H}$ perfluorooctyl- $1 H, 1 H, 2 H, 2 H$-perfluorodecyl)phosphate (6:2/ 8:2 diPAP), $N$-ethyl perfluorooctane sulfonamide ethanolbased phosphate diester (diSAmPAP), $2 \mathrm{H}$-perfluoro-2decenoic acid (8:2 FTUCA), $N$-ethylperfluoro-1octanesulfonamidoacetic acid (EtFOSAA), sodium perfluoro1-octanesulfonate (PFOS), 3-perfluoroheptyl propanoic acid (7:3 PFCA), 2H-perfluoro-2-decenoic acid (8:2 PFCA), and sodium $1 H, 1 H, 2 H, 2 H$-perfluorooctane sulfonate (6:2 FTSA) were obtained from Wellington Laboratories, Inc. (Guelph, Ontario, Canada). Tris $(1 H, 1 H, 2 H, 2 H$-perfluorohexyl) 
phosphate $(6: 2$ polyfluorinated trialkylated phosphate ester, triPAP) was purchased from Chiron AS (Trondheim, Norway). Bis(perfluorohexyl)phosphonic acid (perfluorinated phosphonic acid, PFPIA) was purchased from Toronto Research Chemicals (North York, Ontario, Canada). PFOA was obtained from ABCR (Karlsruhe, Germany). Perfluorooctanesulfonamide (FOSA) was obtained from SynQuest Laboratories (Alachua, FL, USA).

\section{Sample collection and preparation}

The homogenized, freeze-dried soil samples from the contaminated area in Germany were collected in 2017 and provided by the Landwirtschaftliches Technologiezentrum Augustenberg (LTZ) (Karlsruhe, Germany). Sample 1 originated from Hügelsheim and samples 2, 3, and 4 from the area around Mannheim. Five grams of the soil samples was weighed in a $50-\mathrm{mL}$ polypropylene (PP) tube, and $10 \mathrm{~mL}$ $\mathrm{MeOH}$ was added. The mixture was thoroughly vortexed for $2 \mathrm{~min}$, sonicated for $10 \mathrm{~min}$, and then put on a horizontal shaker for $24 \mathrm{~h}$. After centrifuging (10 min, 4000 relative centrifugal force (rcf)), the supernatant was transferred to a 20-mL PP vessel using a glass pipette. The extraction was repeated with $10 \mathrm{~mL} \mathrm{MeOH}$ as described. The supernatants were combined, heated to $40{ }^{\circ} \mathrm{C}$, and evaporated to less than $1 \mathrm{~mL}$ with a gentle stream of nitrogen. Pure $\mathrm{MeOH}$ was used to adjust the volume to $1 \mathrm{~mL}$. The concentrate was transferred into a PP vial and centrifuged (10 $\mathrm{min}, 4000 \mathrm{rcf}$ ) prior to analysis.

\section{Instrumental analysis}

Samples were analyzed by LC-MS using a 1290 HPLC (Agilent Technologies, Waldbronn, Germany) coupled to a 6550 QTOF mass spectrometer (Agilent Technologies, Santa Clara, USA). An Agilent C18 column (Poroshell 120 EC-C18, $2.1 \mathrm{~mm} \times 100 \mathrm{~mm}$, particle size $2.7 \mu \mathrm{m}$ ) was used to separate the analytes, and the flow rate was set to $0.4 \mathrm{~mL} / \mathrm{min}$ and the temperature to $40{ }^{\circ} \mathrm{C}$. Eluent A $\left(95: 5 \mathrm{H}_{2} \mathrm{O} / \mathrm{MeOH}\right)$ and eluent $\mathrm{B}\left(5: 95 \mathrm{H}_{2} \mathrm{O} / \mathrm{MeOH}\right)$, both with $2 \mathrm{mM} \mathrm{NH}_{4} \mathrm{Ac}$, were used for gradient elution. The gradient started with $25 \% \mathrm{~B}$, followed by a linear increase to $85 \% \mathrm{~B}$ within $2 \mathrm{~min}$ and to $100 \% \mathrm{~B}$ within $2.5 \mathrm{~min}$, and kept isocratic for another $12 \mathrm{~min}$. Initial conditions were reset at $12.1 \mathrm{~min}$ with a subsequent equilibration time of $15 \mathrm{~min}$. The injection volume of the sample was $20 \mu \mathrm{L}$. The electrospray ionization (ESI) source, equipped with the Agilent Jet Stream technology, was operated in negative ionization mode. The MS measurements were performed in scan mode for screening and in both targeted MS/MS mode and allion fragmentation (AIF) for further structural information. AIF measurements were performed at collision energies of $0 \mathrm{eV}, 20 \mathrm{eV}$, and $40 \mathrm{eV}$. Instrumental parameters for MS/MS measurements are given in Electronic Supplementary Material (ESM) 1 (section targeted MS/MS measurements).

To validate the extraction method, targeted MS/MS measurements were performed with uncontaminated soil spiked with $10 \mathrm{ng}$ of several PFASs (diSAmPAP, 8:2 PFCA, EtFOSAA, 6:2 FTSA) each. Original uncontaminated and spiked soil samples were extracted in the same way as the contaminated samples 1-4. The results are shown in the ESM (Figs. S1.42 to S1.45).

\section{Data analysis and HS detection}

The recorded data were processed using the molecular feature extraction (MFE) algorithm of the MassHunter software (versions B.07.00 and 10.0; Agilent Technologies). Features were narrowed down to mass defects between 0.25 and $+0.1 \mathrm{Da}$ as well as an intensity of at least 1000 counts. To validate the mass defect filtering, we used the curated Organisation for Economic Co-operation and Development (OECD) PFAS list (list of acronym PFASOECDNA, available from the EPA dashboard) and calculated the mass defect of all entries. Out of a total of 3213 PFASs, 2982 substances had a mass defect between -0.25 and +0.1 , showing that an application of this filter covers $92.8 \%$ of all PFASs within this database. The resulting compounds after filtering are hydrocarbon compounds with chemical formulae containing more or less elements with negative mass defects (e.g., F, Cl, Br, I, O, $\mathrm{P}, \mathrm{S})$.

The selected features were exported as a CSV file which could then be imported to Matlab (MathWorks, Natick, USA) to automatically be processed by FindSeries, a code which was written in-house for Kendrick mass defect (KMD) analysis of PFAS. As proposed in 1963 by Edward Kendrick [37], the KMD was calculated according to Eqs. (1) and (2). By normalizing the observed mass $(\mathrm{OM})$ of a compound by the integer mass of the repeating unit (e.g., 50:49.9968 in the case of $\mathrm{CF}_{2}$ ), all homologue compounds with the same core structure but varying numbers of the repeating unit reveal the same KMD. For example, perfluoroheptanoic acid (PFHpA), PFOA, and perfluorononanoic acid (PFNA) have the exact masses $363.9769,413.9737$, and 463.9705 , respectively. They all bear a carboxylic acid group as a common structural moiety and are only distinguished by the number of the repeating units $\left(\mathrm{CF}_{2}\right.$ with an exact mass of 49.9968). For example, the KMD for PFHpA is $-1.96 \times 10^{-4}$ (Eq. 3) and exactly the same number for PFOA and PFNA or even larger PFCAs like perfluorohexadecanoic acid $\left(\mathrm{C}_{16} \mathrm{HF}_{31} \mathrm{O}_{2}\right)$. 
Detected HS are further visualized by plotting the KMD vs. $\mathrm{m} / \mathrm{z}$ where compounds from the same compound class align horizontally.

$\mathrm{KM}=\mathrm{OM} \cdot \frac{\text { nominal mass (repeating unit) }}{\text { exact mass (repeating unit) }}$

$\mathrm{KMD}=$ nominal $\mathrm{KM}-$ exact $\mathrm{KM}$

$\mathrm{KMD}=364-363.9769 \cdot \frac{50}{49.9968}=1.96 \cdot 10^{-4}$

where $\mathrm{KM}$ is the Kendrick mass, $\mathrm{OM}$ is the accurate observed mass of the compound, and KMD is the Kendrick mass defect.

In step 1, FindSeries further compares the measured accurate masses with a database containing exact masses of known PFASs (curated OECD PFAS list, available online at EPA dashboard) [38] using a tolerance of $3 \mathrm{mDa}$. Matching masses are exported, including the respective name, yielding a list of potentially occurring PFASs in the sample. In step 2, FindSeries performs a Kendrick mass analysis for different repeating units such as $-\mathrm{CF}_{2}-,-\mathrm{CF}_{2} \mathrm{O}-$, or $-\mathrm{C}_{2} \mathrm{~F}_{4} \mathrm{O}$ - using a tolerance of $5 \mathrm{mDa}$. This higher tolerance is chosen in step 2 because unlike in step 1, there is no reference mass value from a database, and all members from one HS need to deviate less than the applied tolerance from one another. The resulting HS are subsequently validated by a manual inspection of the chromatographic peaks (Gaussian peak shape, signal-to-noise ratio $>10$ ) and a systematic retention time (RT) shift. The RT difference between the first members of a HS defines a RT shift, and the following homologues need to be in the same range. With increasing mass, a decreasing retention time shift has to be observed due to the increasing elution strength of the LC gradient. For example, four consecutive members of a HS with RT $3 \mathrm{~min}, 4 \mathrm{~min}, 4.1 \mathrm{~min}$, and $6 \mathrm{~min}$ would be discarded (vastly different time shifts with no clear trend). In turn, four consecutive members of a HS with RT $3 \mathrm{~min}, 3.6 \mathrm{~min}$, $4.1 \mathrm{~min}$, and $4.5 \mathrm{~min}$ would be considered for further evaluation (plausible RT shift trend).

If available, one authentic standard per HS was purchased and used for confirmation of the compound identity. According to the scheme of identification confidence from Schymanski et al. [39], detected PFASs were classified by defined confidence levels. For level 1, the structure was confirmed by an authentic standard based on accurate mass and RT. Level 2a was used for members of a HS if one candidate was successfully confirmed by a reference standard and the other candidates exhibited the previously mentioned systematic mass and RT shifts. For level $2 b$, members of a HS with a systematic RT shift were detected and structures were validated with fragmentation patterns. Single features not belonging to a HS were classified as level 3, and compound identifications were tentatively proposed based on accurate mass and matching with the OECD database.

\section{Results and discussion}

Highly contaminated soil samples were analyzed for PFAS precursors and TPs by LC-QTOF-MS. The procedure of the data evaluation is shown in detail for sample 1. Further results for samples 2, 3, and 4 are presented subsequently using the same work flow. For readability, only integer masses are shown in the result tables. The corresponding accurate masses are shown in Tables S1-S6 (see ESM).

Accurate mass scans showed in total 4837 features in the contaminated soil sample 1, 1940 of those had negative mass defects in the range between -0.25 and $+0.1 \mathrm{Da}$, which typically characterize compounds with a high number of elements with negative mass defects, such as fluorine, chlorine, bromine, oxygen, phosphorus, or sulfur, and with a low number of hydrogen atoms with a positive mass defect. The mass defect filter considerably reduced the dataset, even though not all features with negative mass defects can be attributed to highly fluorinated compounds. Accurate mass data have been evaluated by Kendrick mass analysis for compounds characterized by $\mathrm{HS}$ with $\mathrm{CF}_{2}, \mathrm{CF}_{2} \mathrm{O}$, and $\mathrm{C}_{2} \mathrm{~F}_{4} \mathrm{O}$ repeating units.

\section{Data evaluation of sample 1}

FindSeries revealed the occurrence of $329 \mathrm{HS}$ based on $\mathrm{CF}_{2}$ repeating units (see ESM Table S1). This still includes HS with only two compounds. Reducing this list to HS with at least three individual compounds condensed this dataset to 133 HS (see ESM Table S2). Visualization in a $\mathrm{CF}_{2}$-based Kendrick mass defect plot (Fig. 1) easily allows a first overview on the data. Compounds belonging to the same class are aligned horizontally. As a second criterion, increasing retention time with increasing mass is used to identify members of

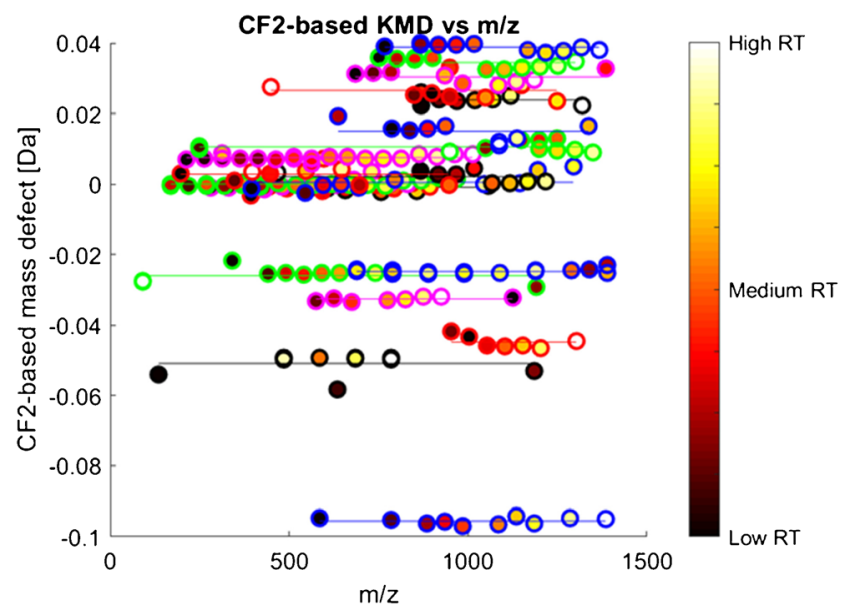

Fig. 1 Kendrick mass defect for $\mathrm{CF}_{2}$ repeating units vs. mass-to-charge ratio. Only selected HS with at least 7 homologues are shown. Each feature is represented by a circle in different colors to distinguish HS. The color code of the dot filling corresponds to the normalized RT and therefore comprises the full range for each HS from dark to white for short to long RT 
a true HS. This is visualized by color code of the data points in Fig. 1 from dark to white for increasing retention times.

Finally, 70 HS fulfilled the criteria of HS detection with at least 3 homologues and systematic RT shifts. They are marked in green in ESM Table S2.

This reduced list of the accurate masses is matched with the OECD database (available in ESM Table S3) for a first tentative identification. For example, HS 82 consists of 20 single features (see ESM Table S1). Plotting these features in a $\mathrm{m} / \mathrm{z}$ vs. RT diagram (see Fig. 2) shows a clear RT shift for most of the features. Four features obviously differ from the clear trend (marked with encircled multiplication sign), and another feature with $\mathrm{m} / \mathrm{z} 413$ at the correct RT is missing (marked with empty circle). The absence of a signal may be due to missing of the compound, low signal intensity, or mismatch in the featurefinding algorithm MFE. All features of this HS could be matched with at least one compound of the OECD PFAS database. The number of matches is indicated in the "Presence" row. The lowest $\mathrm{m} / \mathrm{z}$ in this HS, $\mathrm{m} / \mathrm{z} 212.9793$, was matched with the two isomeric PFCAs heptafluorobutyric acid (CAS number 375-224) and heptafluoroisobutyric acid (CAS number 335-10-4). Also, all other homologues of HS 82 could be assigned to PFCAs.

Next, the extracted ion chromatograms (EICs) of the features were checked manually at a mass accuracy of $10 \mathrm{ppm}$ (ESM Fig. S1). Since the MFE algorithm could always miss single features, also missing members of the HS were included and confirmed, for example the presence of the previously discussed missing feature at $\mathrm{m} / \mathrm{z} 413$. Since some peaks exhibit shoulders or not well separated peaks in front $(\mathrm{m} / \mathrm{z}$ 412.9664), the MFE algorithm registered two peaks which were marked with encircled multiplication sign in Fig. 2. m/ z 412.9664 (also a fronting peak) was integrated once with a

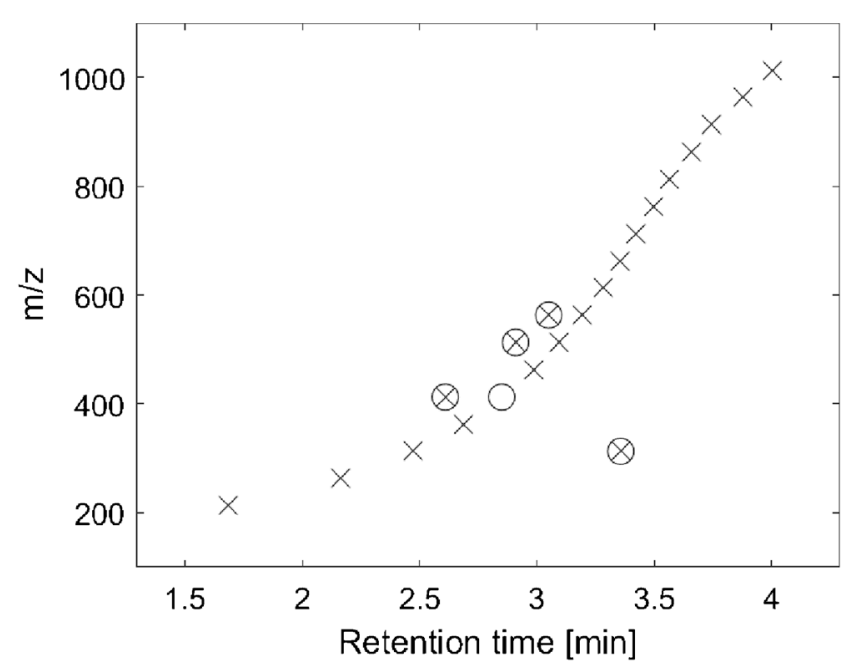

Fig. 2 Systematic retention time shift for HS 82 (PFCAs). Legend: multiplication sign, feature from FindSeries following the RT shift trend; encircled multiplication sign, feature from FindSeries not following the RT shift trend; empty circle, missing feature from FindSeries (possible miss from the MFE) rather short RT and therefore explains the missing feature in Fig. 2. Identification of $\mathrm{m} / \mathrm{z} 412.9664$ as PFOA could be confirmed by an authentic standard (ESM Fig. S1.1). The other PFCA homologues of HS 82 could be identified with confidence level 2a. This procedure was repeated for each of the 70 HS which are marked in green in ESM Table S2. This resulted in the identification of the following substance classes: PFSAs, diSAmPAPs, FTUCAs, diPAPs, and $n: 3$ PFCAs. Identification based on standards for these evaluated substance classes is shown in Figs. S1.2 to S1.8 (see ESM).

Further identifications based on matches with the OECD PFAS database with only one homologue are shown in ESM Table S3. For example, m/z 497.9457 (line 153 in ESM Table S3) was assigned to FOSA and finally confirmed by an authentic standard (ESM Fig. S1.3). Chromatograms were checked manually for further perfluoroalkanesulfonamide (FASA) homologues which were not detected in this case. This procedure was repeated for most compounds in ESM Table S3. Compounds are discarded which are likely to be false positives, for example if no ionization in ESI negative is expected. This procedure further identified the following substance classes: FASA, $N$-methyl perfluoroalkanesulfonamide (MeFASA), $N$-ethyl perfluoroalkanesulfonamide (EtFASA), and $N$-ethylperfluoro-1-alkanesulfonamidoacetic acid (EtFASAA) (one compound each). A complete list of all findings, including the identification level according to Schymanski et al. [39], is presented in Table 1, and the identified substance classes are discussed subsequently.

\section{diPAPs (HS 252) and triPAPs (HS M1)}

A total of 8 diPAP homologues were detected (corresponding to $4: 2 / 6: 2,6: 2 / 6: 2,6: 2 / 8: 2,8: 2 / 8: 2,8: 2 / 10: 2,10: 2 / 10: 2,10: 2 /$ $12: 2,12: 2 / 12: 2$, and $12: 2 / 14: 2$ ) and confirmed by an authentic standard of 6:2/8:2 diPAP. diPAPs are known to be used in food contact paper to act as grease repellant [6], making their occurrence in the paper sludge that was applied on the arable land plausible. Manual inspection of the diPAP chromatograms revealed further peaks with longer retention times. However, these peaks were suspected to originate from triPAPs which are impurities in diPAP products [6]. During ESI, triPAPs are fragmented in-source by the loss of a fluorotelomer alcohol (FTOH) group and therefore appear as diPAPs in LC-MS chromatograms [6, 40, 41]. However, from one triPAP, multiple peaks can result from in-source fragmentation (e.g., 6:2/8:2/10:2 triPAP) depending on which FTOH chain is lost. As a result, an unequivocal identification of triPAPs is challenging, also because authentic standards are not available for all homologues. Therefore, only 6:2/6:2/6:2 triPAP could be identified with an authentic standard, and the other homologues could be tentatively identified and were assigned to confidence level 3 . 


\section{diPAP TPs: FTUCAs (HS 40), n:3 PFCAs (HS 265), and PFCAs (HS 82)}

DiPAPs and triPAPs can be transformed into FTOHs by cleavage of the phosphate ester bonds [42], which can be further degraded to several compound classes like FTUCAs, PFCAs, or $n: 3$ PFCAs [43].

Five homologues of FTUCAs have been detected in sample $1(10: 2,12: 2,14: 2,16: 2$, and 18:2). Based on a study by Liu and Avendano [44], 8:2 FTUCA is a precursor of several PFCAs (perfluorohexanoic, perfluoroheptanoic, and perfluorooctanoic acids). Longer FTUCA homologues are hence expected to transform into longer-chain PFCAs. An authentic standard for 8:2 FTUCA was used to evaluate the mass fragmentation, which is characterized by the loss of $\mathrm{HFCO}_{2}$ at a collision energy of $20 \mathrm{eV}$. The same fragments were observed for the FTUCA congeners that are found in the soil sample (data available in ESM Figs. S1.11-S1.16), and confidence level $2 \mathrm{~b}$ was hence assigned to this HS.

Further degradation of FTUCAs can produce $n: 3$ PFCAs of which five were detected (7:3, 9:3, 11:3, 13:3, and 15:3) and confirmed by a reference standard of 7:3 PFCA. These $n: 3$ PFCAs are suggested to further break down into PFCAs [44]. In total, 16 different PFCA homologues were found, ranging from carbon chain lengths $\mathrm{C}_{5}$ up to $\mathrm{C}_{20}$. PFOA was used as reference standard. PFCAs are rather persistent compounds which will hardly be further degraded in the environment [43].

\section{diSAmPAPs (HS 17)}

Three homologues of the diSAmPAP class $\left(\mathrm{C}_{7} / \mathrm{C}_{8}, \mathrm{C}_{8} / \mathrm{C}_{8}\right.$, and $\mathrm{C}_{8} / \mathrm{C}_{9}$ ) were detected in sample $1 . \mathrm{C}_{8} / \mathrm{C}_{8}$ was confirmed by a standard and is with $98 \%$ of the intensity of all diSAmPAPs by far the most abundant homologue in sample 1. This points to the use of PFAS products on a C8-based chemistry. DiSAmPAP is a chemical that was used directly in food contact papers and had a high production volume until 2002 [31].

\section{diSAmPAP TPs: EtFASAAs (HS M2), MeFASAs (HS M3), EtFASAs (HS M4), FASAs (HS M5), and PFSAs (HS 321)}

The degradation of diSAmPAPs in marine sediments showed several TPs like EtFASAAs, EtFASAs, FASAs, and PFSAs [22], which could be confirmed in our work. MeFASA most likely is an impurity in diSAmPAP products. Identification of diSAmPAPs, EtFASAAs, FASAs, and PFSAs was confirmed by a $\mathrm{C}_{8}$-based reference standard of each compound class.

As already expected from the dominant occurrence of $\mathrm{C}_{8} /$ $\mathrm{C}_{8}$ diSAmPAP, no other homologues than $\mathrm{C}_{8}$ could be detected for EtFASAA, MeFASA, EtFASA, and FASA. The intensity of PFOS was higher than $99.5 \%$ compared to the sum of the intensities from all measured PFSAs. This finding suggests PFOS as the degradation product of $\mathrm{C}_{8} / \mathrm{C}_{8}$ diSAmPAPs.

\section{False positive assignments}

PFPIAs were first suggested to occur in the contaminated soil sample (see ESM Table S1, HS 4). Even though only higher homologues than the available reference standard of the $\mathrm{C}_{6} /$ $\mathrm{C}_{6}$-PFPIA ( $\left.\mathrm{m} / \mathrm{z} 700.9221\right)$ were detected in the soil, the standard was used to record an MS/MS spectrum at $40 \mathrm{eV}$ (ESM Fig. S1.9). Two fragments at $\mathrm{m} / \mathrm{z} 62.9638\left(\mathrm{PO}_{2}\right)$ and $\mathrm{m} / \mathrm{z}$ $400.9415\left(\mathrm{C}_{6} \mathrm{~F}_{14} \mathrm{O}_{2} \mathrm{P}\right)$ have been detected. While the fragment $\mathrm{m} / \mathrm{z} 400.9415$ is dependent on the PFPIA chain length, the $\mathrm{PO}_{2}$ fragment should occur for any PFPIA homologue but did not. Therefore, PFPIA homologues could not be identified (ESM Fig. S1.10).

\section{Further unknown PFASs}

In addition to the identified PFASs, the data still contain many unidentified PFASs occurring in $\mathrm{HS}$ with $\mathrm{CF}_{2}, \mathrm{CF}_{2} \mathrm{O}$, and $\mathrm{C}_{2} \mathrm{~F}_{4} \mathrm{O}$ repeating units. FindSeries suggested $50 \mathrm{HS}$ based on $\mathrm{CF}_{2}$ repeating units, $23 \mathrm{HS}$ based on $\mathrm{CF}_{2} \mathrm{O}$, and $13 \mathrm{HS}$ based on $\mathrm{C}_{2} \mathrm{~F}_{4} \mathrm{O}$, each with a minimum number of 5 individual compounds (data available in ESM Tables S5 and S6). While some of them may be false positives for example due to in-source fragmentation or adduct formation, we expect also the presence of true positives which are absent in the OECD PFAS database and may be degradation products of the original PFAS products.

Table 1 Identified PFAS classes and their number of homologues in sample 1

\begin{tabular}{lllll} 
HS no. & Identified as & $\begin{array}{l}\text { Number of } \\
\text { homologues }\end{array}$ & Mass range & Identification level \\
\hline 252 & diPAPs & 8 & $689-1389$ & Level 2a $(1 \times$ level $1 *)$ \\
M1 & triPAPs & $>4$ & N/A & Level 3 $(1 \times$ level $1 *)$ \\
40 & FTUCAs & 5 & $557-957$ & Level 2b \\
265 & $n: 3$ PFCAs & 5 & $441-841$ & Level 2a $(1 \times$ level $1 *)$ \\
82 & PFCAs & 16 & $263-1013$ & Level 2a $(1 \times$ level $1 *)$ \\
17 & diSAmPAPs & 3 & $1103-1263$ & Level 2a $(1 \times$ level $1 *)$ \\
M2 & EtFASAAs & 1 & 584 & Level 1 \\
M3 & MeFASAs & 1 & 512 & Level 3 \\
M4 & EtFASAs & 1 & 526 & Level 3 \\
M5 & FASAs & 1 & 498 & Level 1 \\
321 & PFSAs & 5 & $399-599$ & Level 2a $(1 \times$ level $1 *)$ \\
\hline
\end{tabular}

Details can be found in ESM Table S1 under the corresponding HS no. Manually detected compound classes are marked with an M. For accurate masses of all homologues, see ESM Table S4

*For reference standards, see ESM Table S4 
Table 2 Identified PFAS classes and their number of homologues in sample 2

HS no. Identified as Number of Mass range Identification level homologues

\begin{tabular}{lllll}
\hline 248 & diPAPs & 7 & $789-1389$ & Level 2a $(1 \times$ level 1) \\
M1 & triPAPs & $>4$ & N/A & Level 3 $(1 \times$ level 1) \\
86 & PFCAs & 17 & $213-1013$ & Level 2a $(1 \times$ level 1) \\
23 & diSAmPAPs & 1 & 1203 & Level 1 \\
M2 & EtFASAAs & 1 & 584 & Level 1 \\
M3 & FASAs & 1 & 498 & Level 1 \\
312 & PFSAs & 7 & $299-599$ & Level 2a $(1 \times$ level 1) \\
166 & FTSAs & 6 & $427-927$ & Level 2a $(1 \times$ level 1) \\
\hline
\end{tabular}

Manually detected compound classes are marked with an M. For accurate masses of all homologues, see ESM Table S4

\section{Results for samples 2, 3, and 4 and comparison of all four samples}

Analogous to sample 1, samples 2, 3, and 4 were evaluated and the results are summarized in Tables 2, 3, and 4. Detailed information and accurate masses are presented in ESM Table S4.

Also samples 2, 3, and 4 are characterized by the presence of diPAPs ( 7 to 8 homologues: 4:2/6:2, 6:2/6:2, 6:2/8:2, 8:2/ $8: 2,8: 2 / 10: 2,10: 2 / 10: 2,10: 2 / 12: 2,12: 2 / 12: 2$, and 12:2/14:2), $\mathrm{C}_{8} / \mathrm{C}_{8}$ diSAmPAP (only 1 dominating homologue), and their TPs. In all four samples, PFCAs reveal a large number of homologues between 14 and 17. $n: 3$ PFCAs were detected only in samples 1 and 3 and FTSAs only in samples 2 and 4. FTSAs are not expected to be TPs of either diPAPs or diSAmPAPs. Hence, they could either occur from the degradation of another, so-far unidentified or already fully degraded, precursor.

Table 3 Identified PFAS classes and their number of homologues in sample 3

HS no. Identified as Number of Mass range Identification level homologues

\begin{tabular}{lllll}
\hline 250 & diPAPs & 8 & $689-1389$ & Level 2a $(1 \times$ level 1) \\
M1 & triPAPs & $>4$ & N/A & Level 3 $(1 \times$ level 1) \\
265 & $n: 3$ PFCAs & 5 & $441-841$ & Level 2a $(1 \times$ level 1) \\
M2 & PFCAs & 15 & $213-963$ & Level 2a $(1 \times$ level 1) \\
16 & diSAmPAPs & 1 & 1203 & Level 1 \\
M3 & EtFASAAs & 1 & 584 & Level 1 \\
M4 & MeFASA & 1 & 512 & Level 3 \\
M5 & FASAs & 1 & 498 & Level 1 \\
M6 & PFSAs & 4 & $399-549$ & Level 2a $(1 \times$ level 1)
\end{tabular}

Manually detected compound classes are marked with an M. For accurate masses of all homologues, see ESM Table S4
Table 4 Identified PFAS classes and their number of homologues in sample 4

HS no. Identified as Number of Mass range Identification level homologues

\begin{tabular}{lllll}
\hline 290 & diPAPs & 7 & $789-1389$ & Level 2a $(1 \times$ level 1) \\
M1 & triPAPs & $>4$ & N/A & Level 3 $(1 \times$ level 1) \\
M2 & PFCAs & 14 & $213-913$ & Level 2a $(1 \times$ level 1) \\
M3 & diSAmPAPs & 1 & 1203 & Level 1 \\
M4 & EtFASAAs & 1 & 584 & Level 1 \\
M5 & FASAs & 1 & 498 & Level 1 \\
M6 & PFSAs & 4 & $399-549$ & Level 2a $(1 \times$ level 1) \\
192 & FTSAs & 4 & $627-927$ & $2 \mathrm{a}$ \\
\hline
\end{tabular}

Manually detected compound classes are marked with an M. For accurate masses of the homologues, see ESM Table S4

\section{Estimated concentration levels}

Based on the response factors of the authentic standards for single PFASs at one concentration level (each at $5 \mu \mathrm{g} / \mathrm{L}$ corresponding to $10 \mu \mathrm{g} / \mathrm{kg}$ soil), concentration levels of these PFASs have been estimated for the soil samples. In soil sample 1, PFOA occurred at about $60 \mu \mathrm{g} / \mathrm{kg}$ (ESM Fig. S1.1), PFOS at $100 \mu \mathrm{g} / \mathrm{kg}$ (ESM Fig. S1.4), EtFOSAA at $100 \mu \mathrm{g} / \mathrm{kg}$ (ESM Fig. S1.5), 6:2/8:2 diPAP at $210 \mu \mathrm{g} / \mathrm{kg}$ (ESM Fig. S1.7), and diSAmPAP at $630 \mu \mathrm{g} / \mathrm{kg}$ (ESM Fig. S1.8). In sample 2, PFOA was found at $250 \mu \mathrm{g} / \mathrm{kg}$ (ESM Fig. S1.46) and 6:2/8:2 diPAP at $20 \mu \mathrm{g} / \mathrm{kg}$ (ESM Fig. S1.47); in sample 3, PFOA was $240 \mu \mathrm{g} / \mathrm{kg}$ (ESM Fig. S1.48) and 6:2/8:2 diPAP $90 \mu \mathrm{g} / \mathrm{kg}$ (ESM Fig. S1.49); in sample 4, PFOA had $90 \mu \mathrm{g} / \mathrm{kg}$ (ESM Fig. S1.50) and 6:2/ $8: 2 \mathrm{diPAP} 70 \mu \mathrm{g} / \mathrm{kg}$ (ESM Fig. S1.51).

Based on these results, diPAPs and PFCAs are suggested to be the major contaminants in all four samples. In sample 1, diSAmPAP and its TPs (PFOS and EtFOSAA) are the dominating contaminants followed by diPAPs and its TPs.

\section{Conclusions}

Paper sludge contaminated with PFAS products caused a complex contamination on agricultural soils, since different not further characterized PFAS products and a considerable number of different TPs contribute to the overall contamination. LC-high-resolution mass spectrometry (LC-HRMS) data revealed the complex contamination. The identification of homologous series (HS) of original compounds and transformation products (TPs) by Kendrick mass analysis and systematic retention time shifts was a successful strategy to confidently identify PFASs also with a very limited number of authentic standards. DiPAPs (7-8 homologues, 4:2/6:2 to 12:2/14:2) and diSAmPAP $\left(\mathrm{C}_{8} / \mathrm{C}_{8}\right)$ have been identified as the major 
contaminants of the soils. These PFASs were also used in products for paper impregnation. All other major compound classes could be linked to known TPs of these products with the only exception of FTSAs. The low availability of authentic standards and suitable database entries especially for TPs mainly limit the identification of numerous unknowns which are still in the HRMS data. The application of the proposed workflow on technical products in original form and after degradation tests is a further promising approach to increase the fraction of identified PFAS and their TPs in environmental samples.

Acknowledgments Open Access funding provided by Projekt DEAL. The authors thank the "Landwirtschaftliches Technologiezentrum Augustenberg" (Karlsruhe) for supplying the soil samples, "Technologiezentrum Wasser (TZW)" (Karlsruhe) for the project coordination, and Selina Tisler and Rebecca Bauer for their valuable support for the data evaluation, and the state of Baden-Württemberg for funding of the project EOFplus (L75 17012).

\section{Compliance with ethical standards}

Conflict of interest The authors declare that they have no conflict of interest.

Open Access This article is licensed under a Creative Commons Attribution 4.0 International License, which permits use, sharing, adaptation, distribution and reproduction in any medium or format, as long as you give appropriate credit to the original author(s) and the source, provide a link to the Creative Commons licence, and indicate if changes were made. The images or other third party material in this article are included in the article's Creative Commons licence, unless indicated otherwise in a credit line to the material. If material is not included in the article's Creative Commons licence and your intended use is not permitted by statutory regulation or exceeds the permitted use, you will need to obtain permission directly from the copyright holder. To view a copy of this licence, visit http://creativecommons.org/licenses/by/4.0/.

\section{References}

1. Buck RC, Franklin J, Berger U, Conder JM, Cousins IT, de Voogt P, et al. Perfluoroalkyl and polyfluoroalkyl substances in the environment: terminology, classification, and origins. Integr Environ Assess Manag. 2011;7(4):513-41.

2. OECD. Toward a new comprehensive global database of per- and polyfluoroalkyl subtances (PFASs). 2018. http://www.oecd.org/ chemicalsafety/risk-management/global-database-of-per-andpolyfluoroalkyl-substances.xlsx. Accessed 22.08.2019.

3. Lang JR, Allred BM, Peaslee GF, Field JA, Barlaz MA. Release of per- and polyfluoroalkyl substances (PFASs) from carpet and clothing in model anaerobic landfill reactors. Environ Sci Technol. 2016;50(10):5024-32.

4. Sinclair W, Kim SK, Akinleye HB, Kannan K. Quantitation of gasphase perfluoroalkyl surfactants and fluorotelomer alcohols released from nonstick cookware and microwave popcorn bags. Environ Sci Technol. 2007;41(4):1180-5.

5. Zabaleta I, Negreira N, Bizkarguenaga E, Prieto A, Covaci A, Zuloaga O. Screening and identification of per- and polyfluoroalkyl substances in microwave popcorn bags. Food Chem. 2017;230: 497-506.

6. Trier X, Granby K, Christensen JH. Polyfluorinated surfactants (PFS) in paper and board coatings for food packaging. Environ Sci Pollut Res. 2011;18(7):1108-20.

7. Fujii Y, Harada KH, Koizumi A. Occurrence of perfluorinated carboxylic acids (PFCAs) in personal care products and compounding agents. Chemosphere. 2013;93(3):538-44.

8. Barzen-Hanson KA, Roberts SC, Choyke S, Oetjen K, McAlees A, Riddell N, et al. Discovery of 40 classes of per- and polyfluoroalkyl substances in historical aqueous film-forming foams (AFFFs) and AFFF-impacted groundwater. Environ Sci Technol. 2017;51(4): 2047-57.

9. Favreau P, Poncioni-Rothlisberger C, Place BJ, Bouchex-Bellomie $\mathrm{H}$, Weber A, Tremp J, et al. Multianalyte profiling of per- and polyfluoroalkyl substances (PFASs) in liquid commercial products. Chemosphere. 2017;171:491-501.

10. Giesy JP, Kannan K. Gobal distribution of perfluorooctane sulfonate in wildlife. Environ Sci Technol. 2001;35(7):1339-42.

11. Dreyer A, Weinberg I, Temme C, Ebinghaus R. Polyfluorinated compounds in the atmosphere of the Atlantic and Southern oceans: evidence for a global distribution. Environ Sci Technol. 2009;43(17):6507-14.

12. Rankin K, Mabury SA, Jenkins TM, Washington JW. A North American and global survey of perfluoroalkyl substances in surface soils: distribution patterns and mode of occurrence. Chemosphere. 2016;161:333-41.

13. Yamashita N, Taniyasu S, Petrick G, Wei S, Gamo T, Lam PKS, et al. Perfluorinated acids as novel chemical tracers of global circulation of ocean waters. Chemosphere. 2008;70(7):1247-55.

14. Cui L, Zhou QF, Liao CY, Fu JJ, Jiang GB. Studies on the toxicological effects of PFOA and PFOS on rats using histological observation and chemical analysis. Arch Environ Contam Toxicol. 2009;56(2):338-49.

15. Shi X, Du Y, Lam PK, Wu RS, Zhou B. Developmental toxicity and alteration of gene expression in zebrafish embryos exposed to PFOS. Toxicol Appl Pharmacol. 2008;230(1):23-32.

16. Bao Y, Deng S, Jiang X, Qu Y, He Y, Liu L, et al. Degradation of PFOA substitute: GenX (HFPO-DA ammonium salt): oxidation with UV/persulfate or reduction with UV/sulfite? Environ Sci Technol. 2018;52(20):11728-34.

17. Gomis MI, Vestergren R, Borg D, Cousins IT. Comparing the toxic potency in vivo of long-chain perfluoroalkyl acids and fluorinated alternatives. Environ Int. 2018;113:1-9.

18. Sun M, Arevalo E, Strynar M, Lindstrom A, Richardson M, Kearns $\mathrm{B}$, et al. Legacy and emerging perfluoroalkyl substances are important drinking water contaminants in the Cape Fear River watershed of North Carolina. Environ Sci Technol. 2016;3(12):415-9.

19. Janda J, Nödler K, Brauch H-J, Zwiener C, Lange FT. Robust trace analysis of polar (C 2-C 8) perfluorinated carboxylic acids by liquid chromatography-tandem mass spectrometry: method development and application to surface water, groundwater and drinking water. Environ Sci Pollut Res. 2019;26(8):7326-36.

20. Yu N, Guo H, Yang J, Jin L, Wang X, Shi W, et al. Non-target and suspect screening of per- and polyfluoroalkyl substances in airborne particulate matter in China. Environ Sci Technol. 2018;52(15): 8205-14.

21. Gremmel C, Frömel T, Knepper TP. Systematic determination of perfluoroalkyl and polyfluoroalkyl substances (PFASs) in outdoor jackets. Chemosphere. 2016;160:173-80.

22. Llorca M, Farré M, Picó Y, Müller J, Knepper TP, Barceló D. Analysis of perfluoroalkyl substances in waters from Germany and Spain. Sci Total Environ. 2012;431:139-50.

23. Hollender J, Schymanski EL, Singer HP, Ferguson PL. Nontarget screening with high resolution mass spectrometry in the environment: ready to go? Environ Sci Technol. 2017;51(20):11505-12. 
24. Schymanski EL, Singer HP, Longree P, Loos M, Ruff M, Stravs MA, et al. Strategies to characterize polar organic contamination in wastewater: exploring the capability of high resolution mass spectrometry. Environ Sci Technol. 2014;48(3):1811-8.

25. Wang Y, Yu N, Zhu X, Guo H, Jiang J, Wang X, et al. Suspect and nontarget screening of per- and polyfluoroalkyl substances in wastewater from a fluorochemical manufacturing park. Environ Sci Technol. 2018;52(19):11007-16.

26. Strynar M, Dagnino S, McMahen R, Liang S, Lindstrom A, Andersen E, et al. Identification of novel perfluoroalkyl ether carboxylic acids (PFECAs) and sulfonic acids (PFESAs) in natural waters using accurate mass time-of-flight mass spectrometry (TOFMS). Environ Sci Technol. 2015;49(19):11622-30.

27. Regierungspräsidium Karlsruhe. PFC-Problematik: Zwischenbilanz und Ausblick. 2018. https://rp.badenwuerttemberg.de/rpk/Abt5/Ref541/PFC/Seiten/pfc buergerinfo. aspx. Accessed 22.08.2019.

28. Brendel S, Fetter E, Staude C, Vierke L, Biegel-Engler A. Shortchain perfluoroalkyl acids: environmental concerns and a regulatory strategy under REACH. Environ Sci Eur. 2018;30(1):9.

29. Biegel-Engler A, Vierke L, Apel P, Fetter E, Staude C. Mitteilungen des Umweltbundesamtes zu per- und polyfluorierten Chemikalien (PFC) in Trinkwasser. Bundesgesundheitsbl Gesundheitsforsch Gesundheitsschutz. 2017;60(3):341-6.

30. Schaider LA, Balan SA, Blum A, Andrews DQ, Strynar MJ, Dickinson ME, Lunderberg DM, Lang JR, Peaslee GF. Fluorinated compounds in US fast food packaging. Environ Sci Technol Lett. 2017; 4: 105-111.

31. Benskin JP, Ikonomou MG, Gobas FA, Begley TH, Woudneh MB, Cosgrove JR. Biodegradation of N-ethyl perfluorooctane sulfonamido ethanol (EtFOSE) and EtFOSE-based phosphate diester (SAmPAP diester) in marine sediments. Environ Sci Technol. 2013;47(3):1381-9.

32. Liu C, Liu J. Aerobic biotransformation of polyfluoroalkyl phosphate esters (PAPs) in soil. Environ Pollut. 2016;212:230-7.

33. Ghisi R, Vamerali T, Manzetti S. Accumulation of perfluorinated alkyl substances (PFAS) in agricultural plants: a review. Environ Res. 2018.

34. Bizkarguenaga E, Zabaleta I, Mijangos L, Iparraguirre A, Fernández LA, Prieto A, et al. Uptake of perfluorooctanoic acid, perfluorooctane sulfonate and perfluorooctane sulfonamide by carrot and lettuce from compost amended soil. Sci Total Environ. 2016;571:444-51.

35. Ministerium für Soziales und Integration (Baden-Württemberg). Erste Ergebnisse der PFC-Blutuntersuchungen im Landkreis Rastatt liegen vor. 2018. https://sozialministerium.badenwuerttemberg.de/de/service/presse/pressemitteilung/pid/ersteergebnisse-der-pfc-blutuntersuchungen-im-landkreis-rastatt-liegenvor/. Accessed 22.08.2019.

36. Ingelido AM, Abballe A, Gemma S, Dellatte E, Iacovella N, De Angelis G, et al. Biomonitoring of perfluorinated compounds in adults exposed to contaminated drinking water in the Veneto region, Italy. Environ Int. 2018;110:149-59.

37. Kendrick E. A mass scale based on $\mathrm{CH} 2=14.0000$ for high resolution mass spectrometry of organic compounds. Anal Chem. 1963;35(13):2146-54.

38. Alygizakis N. PFAS|NORMAN: list of PFAS from the OECD curated by Nikiforos Alygizakis. 2019. https://comptox.epa.gov/ dashboard/chemical lists/pfastrier. Accessed 22.08.2019.

39. Schymanski EL, Jeon J, Gulde R, Fenner K, Ruff M, Singer HP, et al. Identifying small molecules via high resolution mass spectrometry: communicating confidence. Environ Sci Technol. 2014;48(4):2097-8.
40. Gebbink WA, Ullah S, Sandblom S, Berger U. Polyfluoroalkyl phosphate esters and perfluoroalkyl carboxylic acids in target food samples and packaging - method development and screening. Environ Sci Pollut Res. 2013;20(11):7949-58.

41. Trier X, Nielsen NJ, Christensen JH. Structural isomers of polyfluorinated di- and tri-alkylated phosphate ester surfactants present in industrial blends and in microwave popcorn bags. Environ Sci Pollut Res Int. 2011;18(8):1422-32.

42. D'Eon J, Mabury S. Production of perfluorinated carboxylic acids (PFCAs) from the biotransformation of polyfluoroalkyl phosphate surfactants (PAPs): exploring routes of human contamination. Environ Sci Technol. 2007;41(13):4799-805.

43. Wang N, Szostek B, Buck RC, Folsom PW, Sulecki LM, Gannon JT. 8-2 Fluorotelomer alcohol aerobic soil biodegradation: pathways, metabolites, and metabolite yields. Chemosphere. 2009; 75(8):1089-96.

44. Liu J, Avendano SM. Microbial degradation of polyfluoroalkyl chemicals in the environment: a review. Environ Int. 2013;61:98-114.

Publisher's note Springer Nature remains neutral with regard to jurisdictional claims in published maps and institutional affiliations.

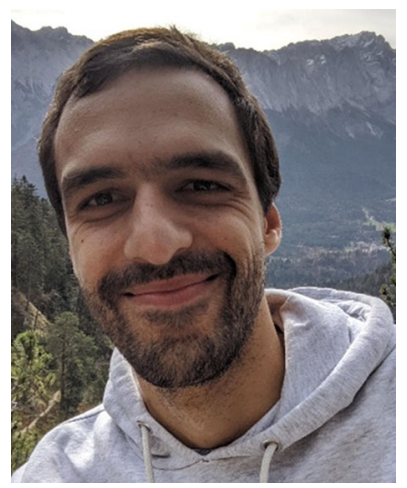

Boris Bugsel is a PhD student in the lab of Christian Zwiener. His research focuses on analysis, occurrence, and environmental fate of per- and polyfluoroalkyl substances (PFASs) and their transformation products. In lab experiments, both photochemical and electrochemical experiments are performed. Transformation products are screened by LC-highresolution mass spectrometry.

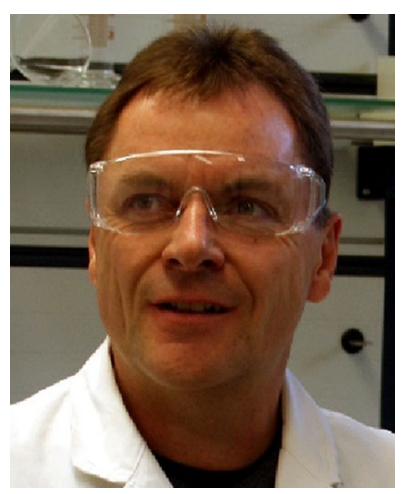

Christian Zwiener is Head of the group of Environmental Analytical Chemistry at the Center for Applied Geoscience, Universität Tübingen. His research focuses on the analysis, occurrence, and fate of organic micropollutants in the environment and in water treatment (e.g., disinfection by-products, pharmaceuticals, personal care products, pesticides, and polyand perfluorinated compounds). This includes the development of analytical approaches for nontarget and suspect screening by LC-high-resolution mass spectrometry, field investigations, and lab-scale experiments for photochemistry, biodegradation, and electrochemical oxidation to identify transformation products. 